\title{
Comparison of image quality and radiation dose using different pre-ASiR-V and post-ASiR-V levels in coronary computed tomography angiography
}

\author{
Yongxia Zhao ${ }^{1}$, Dongxue $\mathrm{Li}^{1}$, Xue Geng ${ }^{1}$, Tianle Zhang ${ }^{2}$, Yize $\mathrm{Xu}^{2}$, and Tianhang Hao ${ }^{1}$ \\ ${ }^{1}$ Affiliation not available \\ ${ }^{2}$ Hebei University
}

May 6, 2020

\begin{abstract}
Objective: To characterize the optimal pre-(ASiR-V and post-ASiR-V for improving image quality and reducing the radiation dose in CCTA. Methods: The study was divided into two parts. In part I, 150 patients for CCTA were prospectively enrolled and randomly divided into five groups (A, B, C, D, and E), with progressive scanning from $40 \%$ to $80 \%$ pre-ASiR-V with $10 \%$ intervals and reconstructing with $70 \%$ post-ASiR-V. The SNR and CNR were calculated. Subjective image quality was assessed using a five-point scale. The radiation dose was recorded and calculated after statistical analysis by optimizing for the best pre-ASiR-V value with the lowest radiation dose while maintaining overall image quality. In part II, the images were reconstructed with the recommended optimal pre-ASiR-V values in part I (D group) was divided into six subgroups (D0-D5 with 40\%-90\% post-ASiR-V and interval 10\%, respectively.).The SNR and CNR of D0-D5 subgroups were calculated and analyzed using one-way analysis of variance, and the consistency of the subjective scores used the k test. Results: There was no significant difference in the SNRs, CNRs, and image quality scores among A, B, C, and D groups. The SNR, CNR, and image quality scores of the E group were lower than those of the A, B, C, and D groups. The mean EDs in the B, C, and D groups were reduced by $7.01 \%, 13.37 \%$, and $18.87 \%$, respectively, when compared with that of the A group. The SNR and CNR of the D4-D5 subgroups were higher than the D0-D3 subgroups, and the image quality scores of the D4 subgroups were higher than the other subgroups. Conclusion: The wide-detector combined with $70 \%$ pre-ASiR-V and $80 \%$ post-ASiR-V significantly reduced the radiation dose of CCTA while maintaining overall image quality compared with the manufacture's recommendation.
\end{abstract}

\section{What does this article add?}

1. In our study, the two models of ASiR-V are explored jointly, and the percentages are divided in detail

2. For the first time, pre ASiR-V and post ASiR-V are applied in CCTA, and the optimal percentage of each mode is explored. To our knowledge, no similar study has been previously reported in the literature for such comparison.

In recent years, multi-slice spiral CT has been rapidly developed for clinical use, and CCTA as a noninvasive and reliable technique has been widely used in the clinic. Because the radiation dose used in the CT examination is associated with the risk of cancer ${ }^{[1]}$, it is important to reduce the patient's radiation dose while retaining the image quality and diagnostic accuracy ${ }^{[2-5]}$. Traditional methods to reduce the radiation dose of CCTA include lowering the tube voltage and the use of tube current automatic modulation technology ${ }^{[6-8]}$. However, the reduction of tube voltage and tube current not only reduces the radiation dose, but it also increases the image noise and has a negative impact on image quality ${ }^{[9,10]}$. So reducing the radiation dose without affecting the diagnosis has been intensely investigated ${ }^{[11,12]}$. ASiR-V is a reconstruction technique that enables reduction in image noise (standard deviation), streak artifact at low signal condition and 
improvement in LCD (Low Contrast Detectability), while preserving the structure details in the image. ASiR-V can be used to reduce the image noise and streak artifact in diagnostic images and thereby reduce the dose required for routine imaging. The scanner allows radiologists to select percentages of ASiR-V. These "percentages" provide a varying degree of noise removal from the images. Different percentages of pre-ASiR$\mathrm{V}$ scanning can modulate the radiation dose and change the image quality by altering the tube current. The purpose of our study was therefore to characterize the wide-detector combined with the best percentage of pre-ASiR-V and post-ASiR-V to improve the image quality and reduce the radiation dose during CCTA examinations, and to optimize the percentages of pre-ASiR-V and post-ASiR-V, without affecting the quality of the clinical diagnosis.

\section{METHODS AND MATERIALS}

\section{Patient population}

This study was approved by our institutional review board, and written informed consent was obtained by all patients. We prospectively enrolled 150 patients (mean age: $53.67 \pm 5.33$ years, range: $39-79$ years; body mass index: $25.3 \pm 2.51 \mathrm{~kg} / \mathrm{m}^{2} ; 71$ females and 79 males) who underwent CCTA from June to December of 2019. Clinical exclusion criteria included allergy to iodine contrast, pregnancy, heart failure, kidney failure and metal implants in the scanning region. All patients were randomly divided into five groups (A, B, C, D, and E), with 30 patients in each group (Table 1 ).

\section{CT protocol}

The study was divided into two parts. In part I, the patients of groups A, B, C, D, and E were treated with progressive scanning from $40 \%$ to $80 \%$ pre-ASiR-V with $10 \%$ intervals, reconstructed with $70 \%$ post-ASiR-V, and optimized for the best pre-ASiR-V value with the lowest radiation dose while maintaining overall image quality. In part II, the images were reconstructed with the recommended optimal pre-ASiR-V value from part I (D group), and 40\%-90\% of post-ASiR-V. The reconstruction group (Dgroup) was divided into six subgroups (interval 10\%, D0: 40\% post-ASiR-V, D1: 50\% post-ASiR-V, D2: $60 \%$ post-ASiR-V, D3: $70 \%$ post-ASiR-V, D4: $80 \%$ post-ASiR-V, and D5: $90 \%$ post-ASiR-V).

All patients were analyzed using the Revolution CT scanner (GE Healthcare, Waukesha, WI, USA). All patients were in the supine position with their hands on top of their heads. Breathing techniques were explained and practiced with the patient before the patients in groups A-E were scanned using pre-ASiR-V from $40 \%$ to $80 \%$ with $10 \%$ ASiR-V intervals. The scan mode was cardiac. The parameters were as follows: the tube voltage was $120 \mathrm{kVp}$, a three-dimensional Smart $\mathrm{mA}$ modulation technique was used, the $\mathrm{mA}$ ranged from $150 \mathrm{~mA}$ to $700 \mathrm{~mA}$, the Gantry rotation time was $0.28 \mathrm{~s}$, the noise index was 9 at a thickness of 2.5 $\mathrm{cm}$, and the scan field of view was $19.2 \mathrm{~cm}$.

For patients with a stable heart rate $(<70$ beats $/$ min), acquisition of the ECG phase of the CCTA scan protocol was set to a $75 \% \mathrm{R}-\mathrm{R}$ interval, if the heart rate of patients was between $70-85$ beats $/ \mathrm{min}$, the scan protocol was set to $30 \%-50 \% \mathrm{R}-\mathrm{R}$ intervals. When the heart rate exceeded $90 \mathrm{beats} / \mathrm{min}$ or there was arrhythmia, 40\%-95\% R-R intervals were used. The contrast medium (Iopamidol, $370 \mathrm{mg} / \mathrm{mL}$, Bracco, Munroe Township, NJ, USA) was injected at $5.0 \mathrm{~mL} / \mathrm{s}$ into the right antecubital vein (median volume: 53 $\mathrm{mL}$; range: $45-67 \mathrm{~mL})$, followed by a saline flush $(40 \mathrm{~mL})$ at the same rate using a high pressure injector (Missouri XD2001; Ulrich, Ulm, Germany). Bolus-tracking technology was used, and the region of interest (ROI: $6 \mathrm{~mm}^{2}$ ) was placed in the center of the ascending aorta with a threshold value of 280 Hounsfield units and a $2 \mathrm{~s}$ delay. The scan range was from $120 \mathrm{~mm}-160 \mathrm{~mm}$ below the tracheal carina to $0.5 \mathrm{~cm}$ from the bottom of the heart.

\section{Objective image quality evaluation}

All images with $0.625 \mathrm{~mm}$ thickness were transferred to the GE AW4.7 workstation for analyses and measurements. The ROI $\left(5 \mathrm{~mm}^{2}\right)$ was placed at the root of the aorta, the left coronary artery, the right coronary artery, and the chest wall musculature at the same level. The clone function of comparison was adopted to ensure the consistency of size and shape of the ROI. The CT attenuation value and Standard Deviation (SD) 
of each ROI was measured. The mean $\mathrm{CT}$ attenuation values and mean SD values from three blood vessels were respectively calculated as CT1 and SD1, we used the value from the chest wall as the CT2 and SD2, with the following calculations: SNR $(\mathrm{SNR}=\mathrm{CT} 1 / \mathrm{SD} 1)$ and $\mathrm{CNR}(\mathrm{CNR}=\mathrm{CT} 1-\mathrm{CT} 2 / \mathrm{SD} 1)^{[13]}$.

\section{Subjective image quality evaluation}

All images were assessed independently with a five-grade scale ${ }^{[14]}$ by two cardiac radiologists who had 10 years of experience in CCTA, and who were blinded to the CT scan parameters. Image scores [?] 3 were considered to be in accordance with the diagnostic requirements. The scoring criteria are shown inTable 2 .

\section{Radiation dose}

The CTDI ${ }_{\text {vol }}$ and DLP were recorded from the CT, and the ED was calculated using the formula: ED = DLP $\mathrm{x} \mathrm{K}$, where $\mathrm{K}$ represented the radiological protection conversion factor, which was the adult heart (chest) $\mathrm{K}=0.026 \mathrm{mSv}^{*} \mathrm{mGy}^{-1} \mathrm{~cm}^{-1[15]}$.

\section{Statistical analyses}

All quantitative data were analyzed with SPSS statistical software for Windows, version 21.0 (SPSS, Chicago, IL, USA) and variables are expressed as the mean +- standard deviation (SD). One-way analysis of variance was used to compare the difference among each group (A-E groups) and each subgroup (D0-D5 subgroups). The variables were as follows: patient age, body mass index (BMI), image noise, CT attention, SNR, CNR, and radiation dose. The $x$ test was used to analyze the consistency of subjective scores for image qualities between the two radiologists (the range of $\mathrm{k}$ values: poor: $0-0.2$, average: $0.21-0.40$, medium: $0.41-0.60$, good: $0.61-0.80$, and excellent: $0.8-1.0)$. A value of $\mathrm{P}<0.05$ was considered statistically significant.

\section{RESULTS}

\section{The general situation}

All patients completed the CCTA examination successfully. The results of our study showed that there was no significance difference in patient age and BMI among the five groups $(\mathrm{P}>0.05$; Table 1$)$.

\section{Quantitative image analysis}

Part I: There was no significant difference in SNR and CNR among the groups A, B, C, and D (SNR: 18.91 $\pm 1.04,18.73 \pm 0.99,18.58 \pm 1.45$, and $18.36 \pm 1.32$, respectively; and CNR: $15.39 \pm 0.88,15.34 \pm 0.81$, $14.98 \pm 1.29$, and $14.87 \pm 1.00$, respectively; $\mathrm{P}>0.05)$. The SNRs and CNRs of group E were lower than those in groups A, B, C, and D ( $\mathrm{P}<0.001)$, (Table 1, Figure 1 ,Figure2 ). Part II: The SNRs of D0-D5 were $20.08 \pm 3.92,21.02 \pm 4.31,22.15 \pm 4.30,19.17 \pm 4.27,24.89 \pm 5.38$, and $26.51 \pm 5.71$, respectively; and the CNRs of D0-D5 were $15.62 \pm 3.51,16.41 \pm 3.84,17.28 \pm 3.80,14.96 \pm 3.82,19.41 \pm 4.90$, and $19.43 \pm 4.65$, respectively. The SNRs and CNRs of the D4 and D5 subgroups were higher than the other subgroups (D0-D3, P <0.05) (Table 3 ,Figure 3 ).

\section{Subjective image analysis}

All images were assessed by two radiologists who had a good subjective consistency $(\mathrm{k}=0.79)$. Part I: There was no significant statistical difference in scores of the subjective image qualities among the four groups (A: $3.73 \pm 0.67, \mathrm{~B}: 3.70 \pm 0.44, \mathrm{C}: 3.68 \pm 0.56$, and $\mathrm{D}: 3.76 \pm 0.50 ; \mathrm{P}>0.05)$, but the scores of subjective image quality for group E $(3.02 \pm 0.69)$ were lower than those of other groups $(\mathrm{P}<0.01)$ (Table $\mathbf{1}$, Figure 1 ). Part II: The scores of subjective image qualities in the D0-D5 subgroups were $3.50 \pm 0.71,3.70 \pm 0.68$, $3.80 \pm 0.75,3.83 \pm 0.69,4.09 \pm 0.83$, and $3.72 \pm 0.51$, respectively. There were significant differences in subgroup D4, whose subjective score was higher than other subgroups $(\mathrm{P}<0.05)$ (Table 3, Figure3 ).

\section{Radiation dose estimations}

The ED of the A-E groups were $15.26 \pm 0.42,14.19 \pm 0.49,13.22 \pm 0.48,12.38 \pm 0.60$, and $11.25 \pm 0.46$, respectively. The percentages of pre-ASiR-V and ED values had a negative correlation, The correlation coefficients $\mathrm{r}=-0.938(\mathrm{P}=0.000)$. Statistical analyses showed that the EDs showed significant differences among the five 
groups $(\mathrm{P}<0.05)$. Compared with group $\mathrm{A}$, the EDs in groups $\mathrm{B}, \mathrm{C}$, and D decreased by $7.01 \%, 13.37 \%$, and $18.87 \%$, respectively (Table 1, Figure 2 ).

\section{Discussion}

With the rapid development of computed tomography (CT), coronary CT angiography (CCTA) has been widely used in the diagnosis of coronary heart disease ${ }^{[16,17]}$. However, the higher radiation dose from the CCTA examination is a concern of radiologists ${ }^{[18,19]}$. ASiR-V is the third generation of iterative reconstruction that can obtain better image quality at the same scanning conditions, when compared with the first generation of $\mathrm{ASiR}^{[20-23]}$, and can reconstruct images more effectively compared with the Model Based Iterative Reconstruction ${ }^{[24,25]}$. It is the reconstruction technique that enables reduction in image noise, streak artifacts at low signal conditions, and improvement in low contrast detectability, while preserving the structural details in the image. ASiR-V can also automatically adjust the scanning $\mathrm{mA}$ according to different tissue densities of the body, to further reduce the radiation dose ${ }^{[26,27]}$. ASiR-V has two modes: pre-ASiR-V and post-ASiR-V. Both of these modes have different options; pre-ASiR-V can adjust the tube current according to automatic tube current modulation technology while the CT value is not affected. Post-ASiR-V can significantly reduce the image noise and streak artifacts to improve image detail and quality. Previous studies have reported that post-ASiR-V with a higher percentage results in an improved image over smooth images, which affect the detection of small lesions ${ }^{[28-30]}$. Choosing an appropriate percentage of post-ASiR-V is therefore a key to maximizing image quality.

Part I of our study showed that the image noise values of all images had a positive correlation with the pre-ASiR-V level, and the SNR and CNR of all images had a negative correlation with the pre-ASiR-V level. The radiation dose of CCTA was reduced with an increase of the pre-ASiR-V levels. There were no significant statistical differences in scores of subjective image quality, SNR, and CNR when the percentage ranges of the pre-ASiR-V level were $40 \%-70 \%$. The image noise significantly increased while scores of subjective image quality, SNR, and CNR were reduced relative to when the percentage range of the pre-ASiR-V level was > $70 \%$. To avoid failure of the CCTA examination, the maximum percentage of pre-ASiR-V levels used was $80 \%$ in our study. Our study also showed that the wide-detector combined with a $70 \%$ pre-ASiR-V level resulted in a lower radiation dose than the value of the manufacturer's recommended value (40\%), which decreased by $18.87 \%$ while retaining image quality.

Part II of our study showed that the image noise value of all images had a negative correlation with the percentage of the post-ASiR-V value, and the SNR and CNR of all images had a positive correlation with the percentage of post-ASiR-V values. The image noise decreased and the SNR and CNR values increased as the percentage of post-ASiR-V level increased. The mean CT attention did not change significantly as the percentage of post-ASiR-V levels increased. The CNR of the reconstructed image was $19.41+-4.90$ when the percentage of post-ASiR-V was $80 \%$. The subjective score of the D0 ${ }^{\sim} 4$ subgroup reconstructed image increased gradually and the D5 subgroup images decreased. It was possible that a high percentage of ASiR-V (90\%) changed the noise component, resulting in an improved image over the smooth method, which affected the diagnosis. In this section, the subjective score of the reconstructed image was the highest with $80 \%$ post-ASiR-V ${ }^{[31]}$.

Our study had some limitations. First, the patient population was relatively small and a certain amount of samples were needed to ensure that the results were accurate. Second, the effects of different heart rates and rhythms on image quality were not considered. Third, we did not assess the effect of different BMIs in this study. In future studies, patients should therefore be grouped according to their BMI to reduce the effects of image quality.

In conclusion, increasing the percentage of pre-ASiR-V significantly reduced the radiation dose. Increasing the percentage of post-ASiR-V also significantly reduced the image noise and improved the image quality. Overall, our study showed that $70 \%$ pre-ASiR-V and $80 \%$ post-ASiR-V was the best reconstruction algorithm in CCTA examinations of adults.

Table 1. The comparison of patients characteristics, image quality evaluation and ED among 
the five groups

\begin{tabular}{|c|c|c|c|c|c|c|}
\hline \multicolumn{2}{|l|}{ Parameters } & A & B & $\mathbf{C}$ & $\mathbf{D}$ & $\mathbf{E}$ \\
\hline \multicolumn{2}{|l|}{$\mathrm{BMI}(\mathrm{kg} / \mathrm{m} 2)$} & $25.79 \pm 3.20$ & $25.43 \pm 2.10$ & $25.61 \pm 3.37$ & $25.62 \pm 2.70$ & $25.63 \pm 2$ \\
\hline \multicolumn{2}{|l|}{ Age (Year) } & $58.21 \pm 10.63$ & $65.70 \pm 12.94$ & $58.70 \pm 11.57$ & $62.90 \pm 11.09$ & $60.48 \pm 1$ \\
\hline \multicolumn{2}{|l|}{ SD } & $28.18 \pm 1.89$ & $28.21 \pm 2.11$ & $28.37 \pm 1.90$ & $28.78 \pm 1.84$ & $30.35 \pm 1$ \\
\hline \multicolumn{2}{|l|}{ SNR } & $18.91 \pm 1.04$ & $18.73 \pm 0.99$ & $18.58 \pm 1.45$ & $18.36 \pm 1.32$ & $17.49 \pm 0$ \\
\hline \multicolumn{2}{|l|}{ CNR } & $15.39 \pm 0.88$ & $15.34 \pm 0.81$ & $14.98 \pm 1.29$ & $14.87 \pm 1.00$ & $14.27 \pm 0$ \\
\hline \multicolumn{2}{|c|}{ Subjective image quality score } & $3.73 \pm 0.67$ & $3.70 \pm 0.44$ & $3.68 \pm 0.56$ & $3.76 \pm 0.50$ & $3.02 \pm 0.6$ \\
\hline \multicolumn{2}{|l|}{$\mathrm{ED}(\mathrm{mSv})$} & $15.26 \pm 0.42$ & $14.19 \pm 0.49$ & $13.22 \pm 0.48$ & $12.38 \pm 0.60$ & $11.25 \pm 0$ \\
\hline Grading score & Noise & \multicolumn{2}{|c|}{ Vessel contrast } & sharpness & \multicolumn{2}{|l|}{$\begin{array}{l}\text { Diagnostic } \\
\text { confidence }\end{array}$} \\
\hline 5 & Very little & \multicolumn{2}{|c|}{ Very clear } & Sharpest & \multicolumn{2}{|c|}{ Fully diagnostic } \\
\hline 4 & Mild & \multicolumn{2}{|l|}{ Clear } & $\begin{array}{l}\text { Better than three, } \\
\text { poorer than five }\end{array}$ & \multicolumn{2}{|c|}{ Good diagnostic } \\
\hline 3 & Moderate & \multicolumn{2}{|c|}{ Less clear } & $\begin{array}{l}\text { Minor burring in a } \\
\text { acceptable image }\end{array}$ & \multicolumn{2}{|l|}{ Diagnostic } \\
\hline 2 & Obvious & \multicolumn{2}{|c|}{ Unclear } & Poorer than three & \multicolumn{2}{|l|}{$\begin{array}{l}\text { Affecting } \\
\text { diagnosis }\end{array}$} \\
\hline 1 & Severe & \multicolumn{2}{|c|}{$\begin{array}{l}\text { Cannot be } \\
\text { displayed }\end{array}$} & Blurry & \multicolumn{2}{|c|}{ Non-diagnostic } \\
\hline
\end{tabular}

Table 3. The comparison of objective parameters and subjective image quality with different post-ASiR-V levels

\begin{tabular}{llll}
\hline Group & SNR & CNR & Image quality score \\
D0 & $20.08 \pm 3.92$ & $15.62 \pm 3.51$ & $3.50 \pm 0.71$ \\
D1 & $21.02 \pm 4.31$ & $16.41 \pm 3.84$ & $3.70 \pm 0.68$ \\
D2 & $22.15 \pm 4.30$ & $17.28 \pm 3.80$ & $3.80 \pm 0.75$ \\
D3 & $19.17 \pm 4.27$ & $14.96 \pm 3.82$ & $3.83 \pm 0.69$ \\
D4 & $24.89 \pm 5.38$ & $19.41 \pm 4.90$ & $4.09 \pm 0.83$ \\
D5 & $26.51 \pm 5.71$ & $19.43 \pm 4.65$ & $3.72 \pm 0.51$ \\
$P$ Value & 0.00 & 0.00 & 0.00 \\
$F$ Value & 11.163 & 11.002 & 205.378 \\
\hline
\end{tabular}




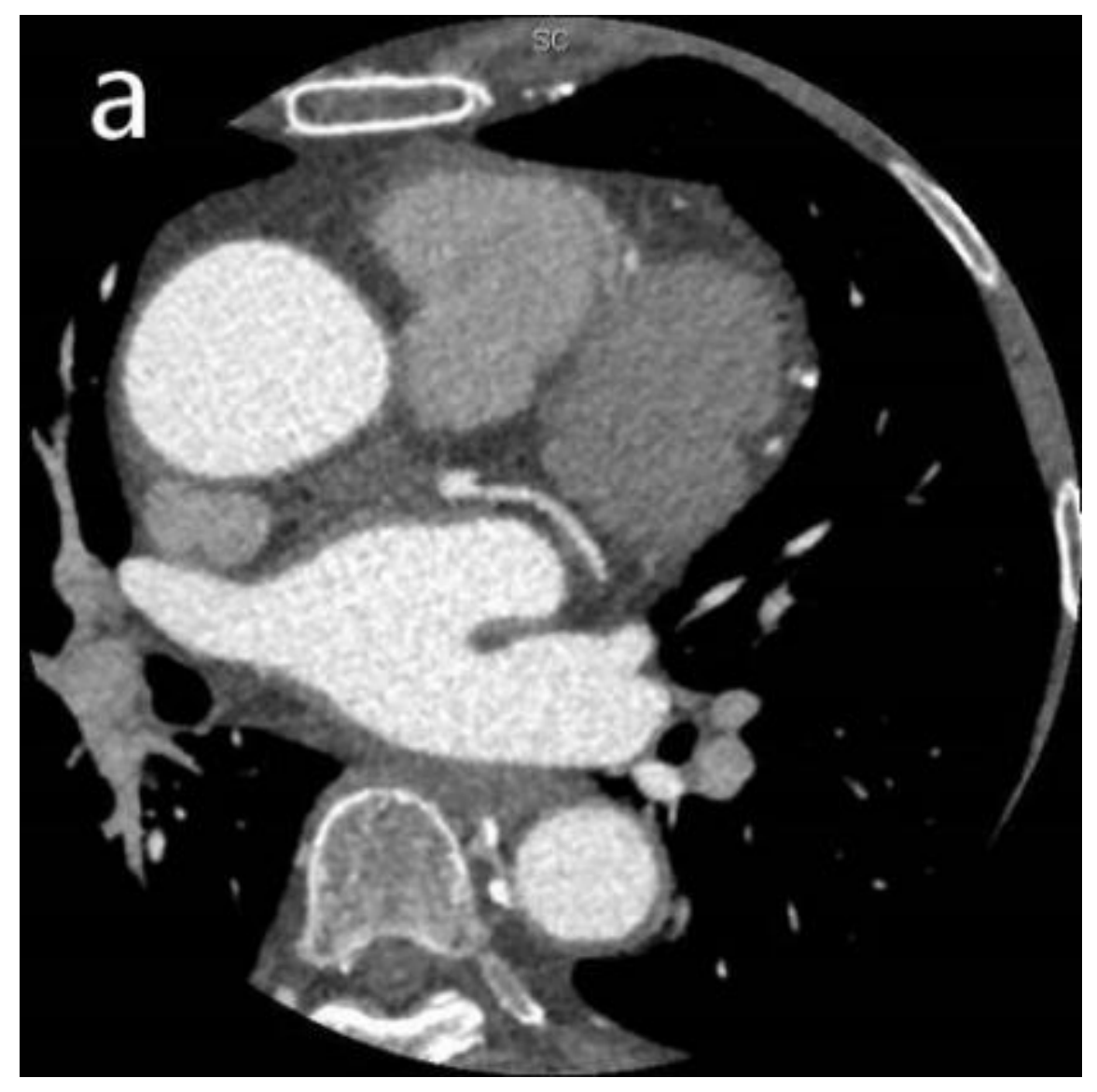




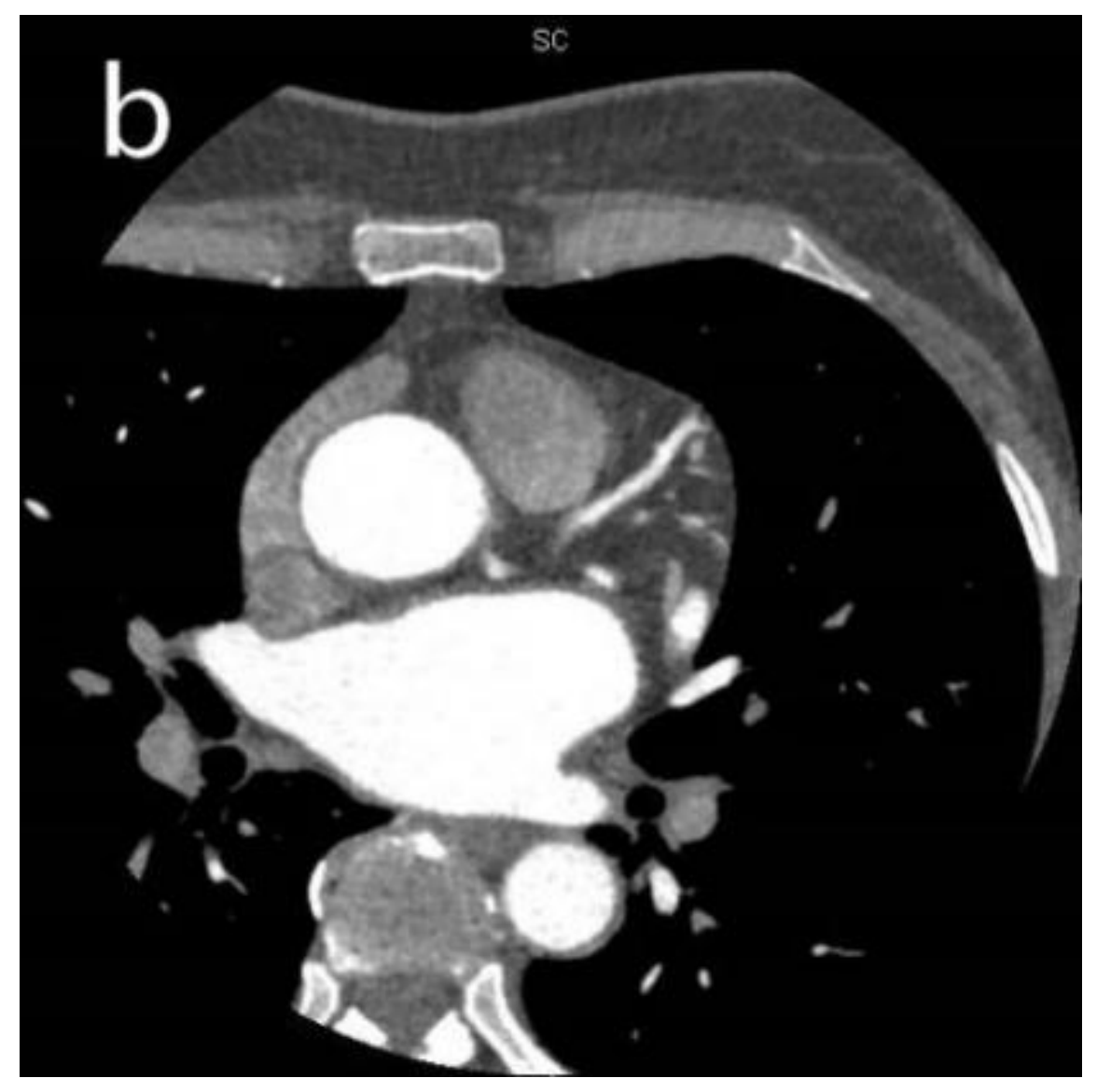




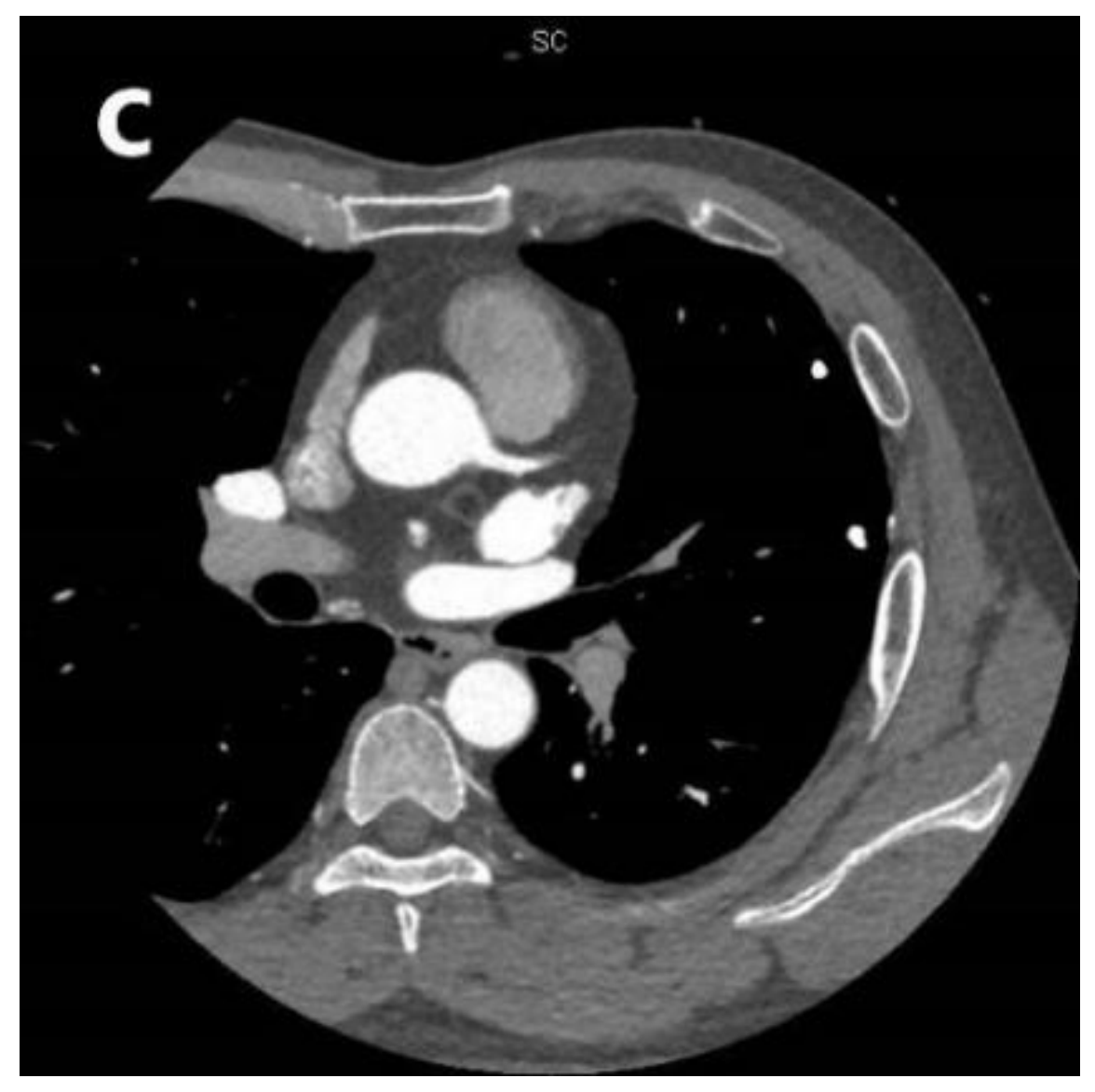




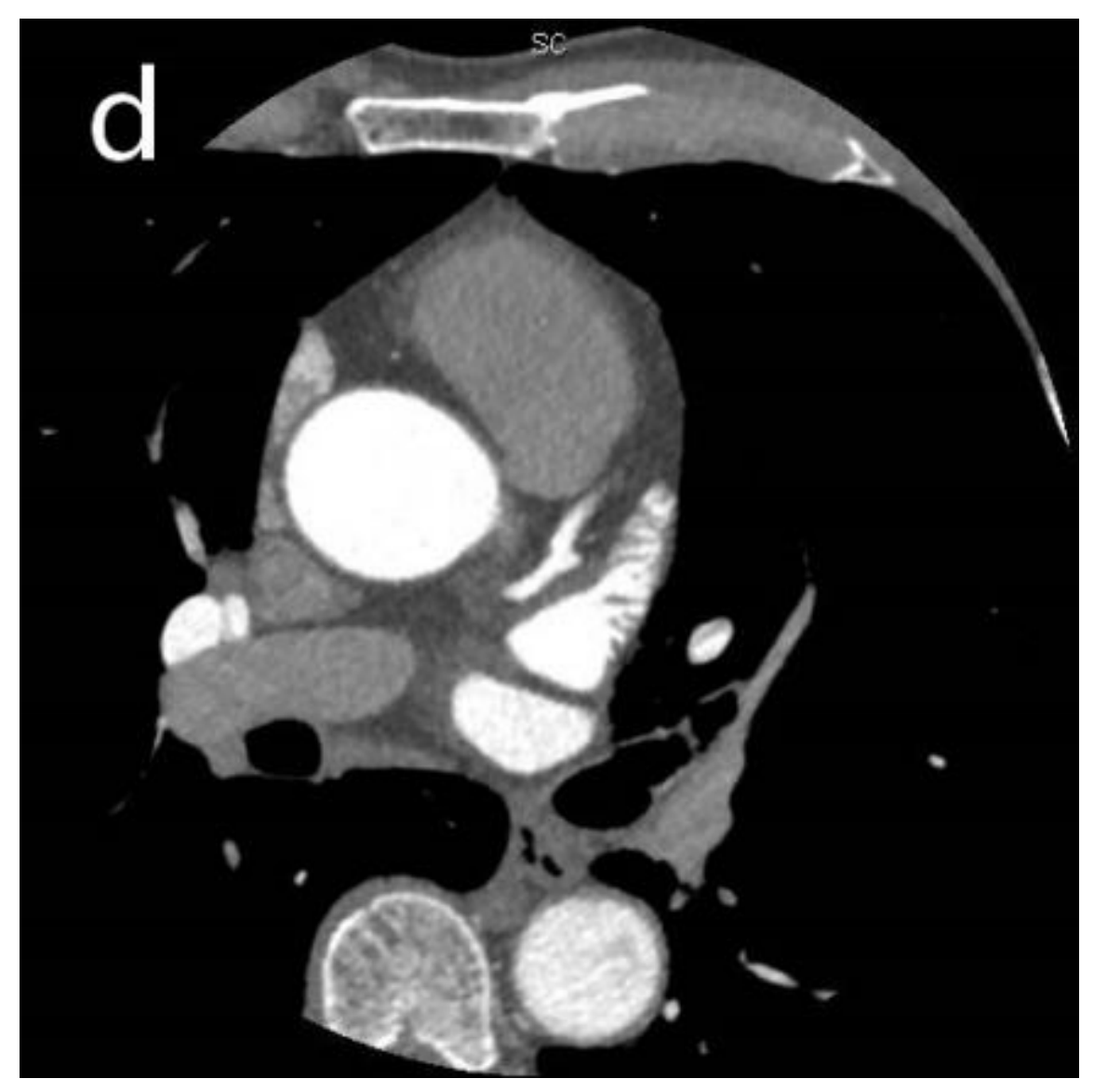




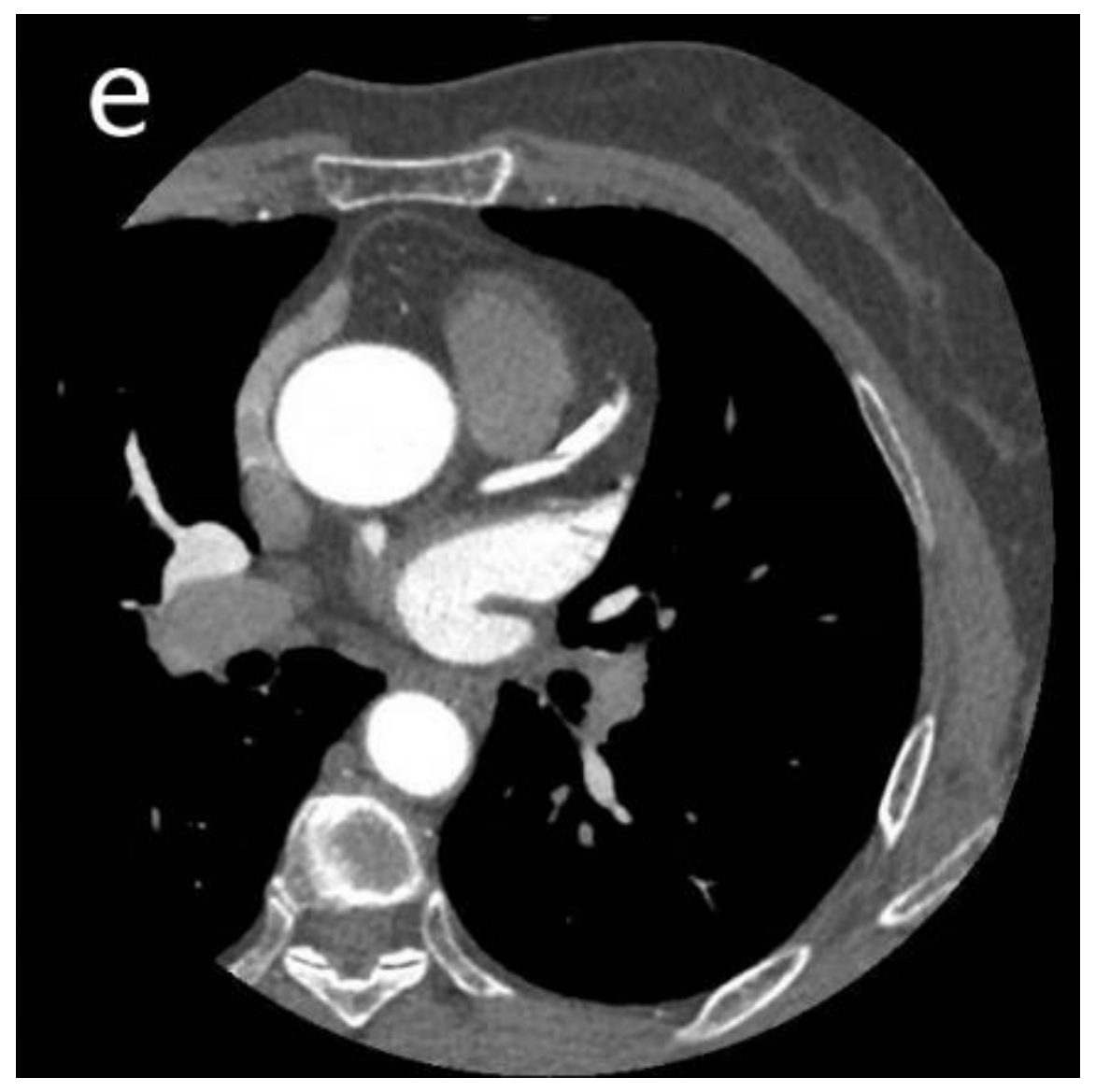

Figure 1. Coronary computed tomography angiography (CCTA) images acquired by the wide-detector combined with different percentages of pre-ASiR-V. The images were scanned with $40 \%$ pre-ASiR-V(a), $50 \%$ pre-ASiR-V(b), $60 \%$ pre-ASiR-V(c), $70 \%$ pre-ASiR-V(d), and $80 \%$ pre-ASiR-V(e) and reconstructed with $70 \%$ post-ASiR-V. The CNR of images (a-e) were 15.35, 15.40, 14.99, 15.29, and 14.34, respectively. The subjective scores of images (a-e) were 3.59, 3.66, 3.73, 3.79, and 3.21, respectively. The effective doses of the patients (a-e) were 8.21, 7.64, 7.15, 6.67, and $6.06 \mathrm{mSv}$, respectively. 


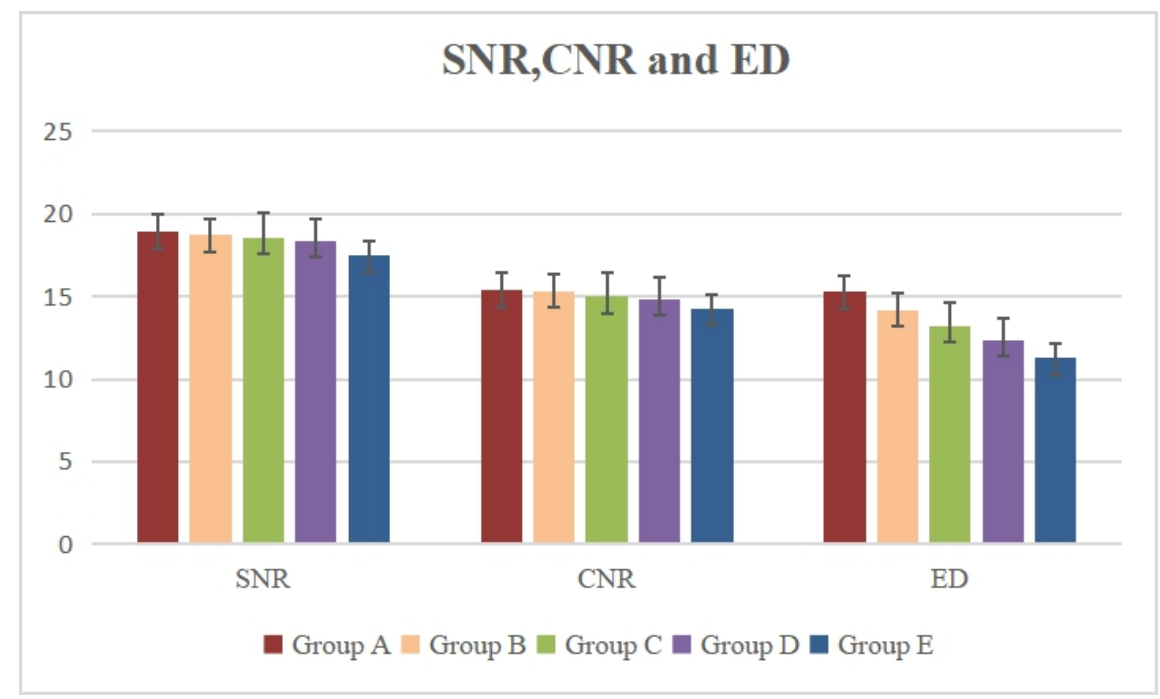

Figure 2. Graph showing the comparisons of SNR, CNR, and ED among different percentages of pre-ASiR-V. Group E showed a lower SNR and CNR than did groups A, B, and C (all $\mathrm{P}<0.05$ ) in all patients, whereas there was no statistically significant difference among groups $\mathrm{A}, \mathrm{B}, \mathrm{C}$, and D ineither SNR $(\mathrm{P}=0.000)$ or CNR $(\mathrm{P}=0.000)$. There were significant differences in $\mathrm{ED}$ among groups $\mathrm{A}, \mathrm{B}, \mathrm{C}$, and $\mathrm{D}(\mathrm{P}=0.000)$. Compared with group A, the EDs in groups B, C, and D decreased by $7.01 \%, 13.37 \%$, and $18.87 \%$, respectively.

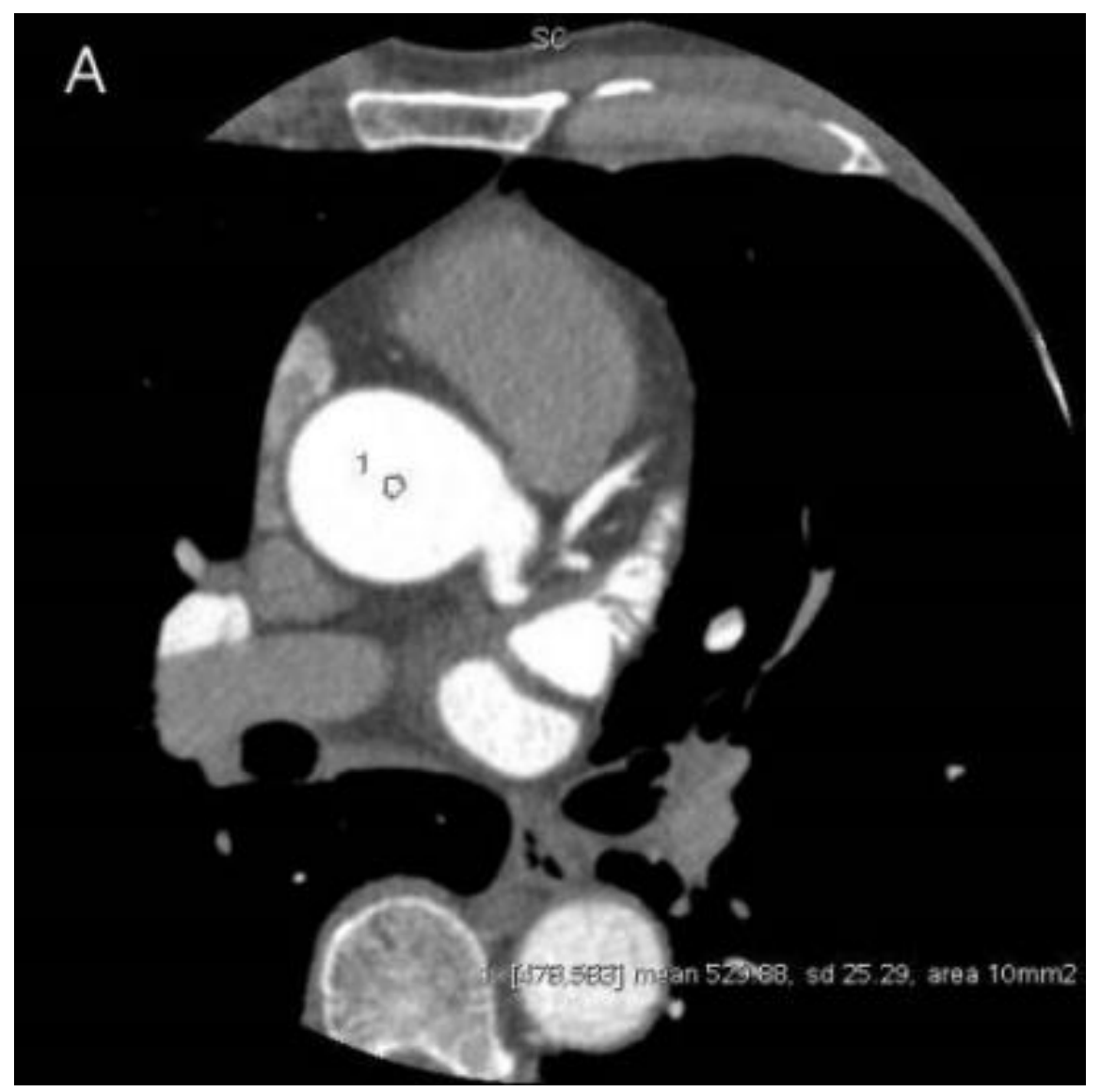




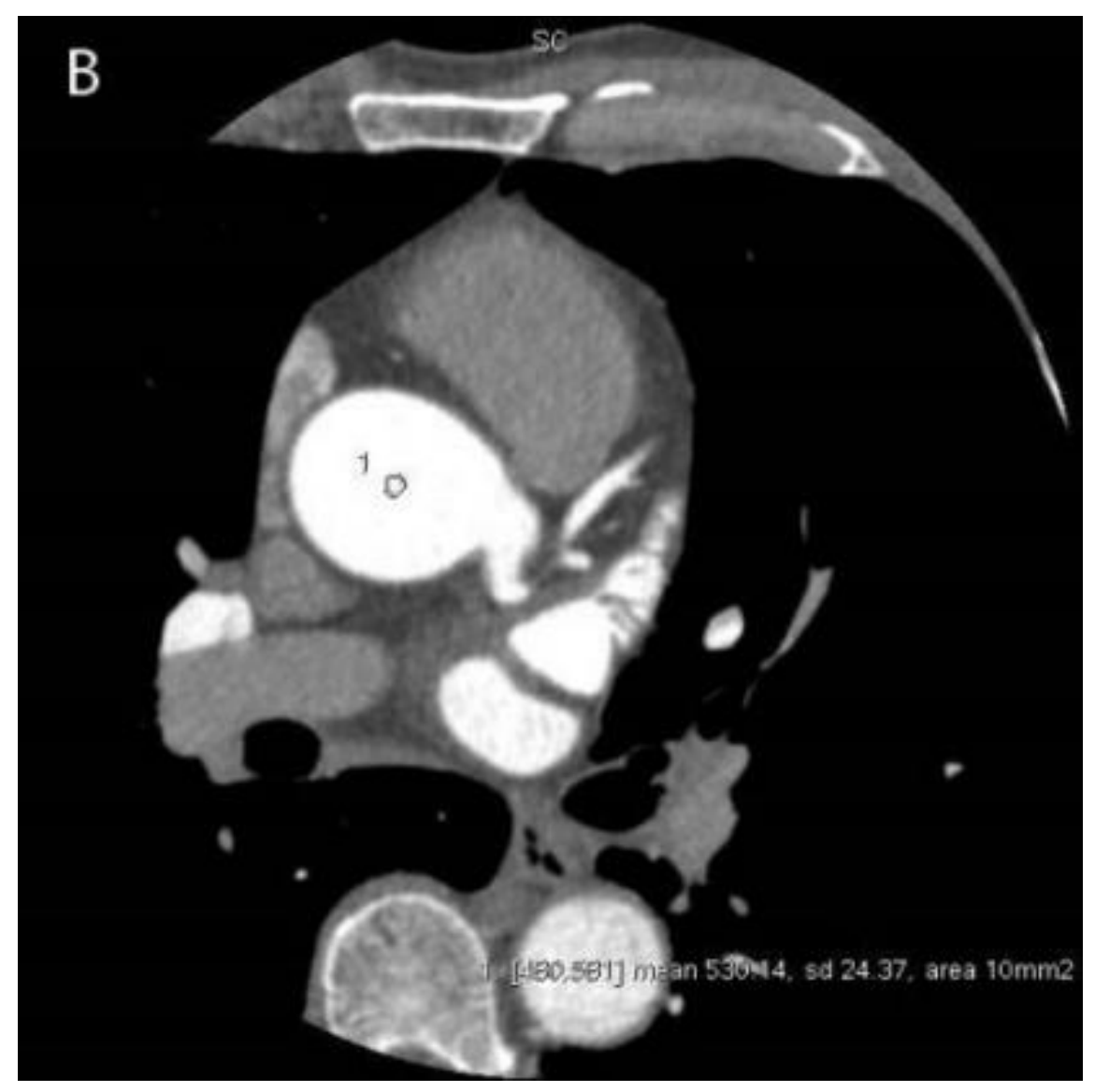



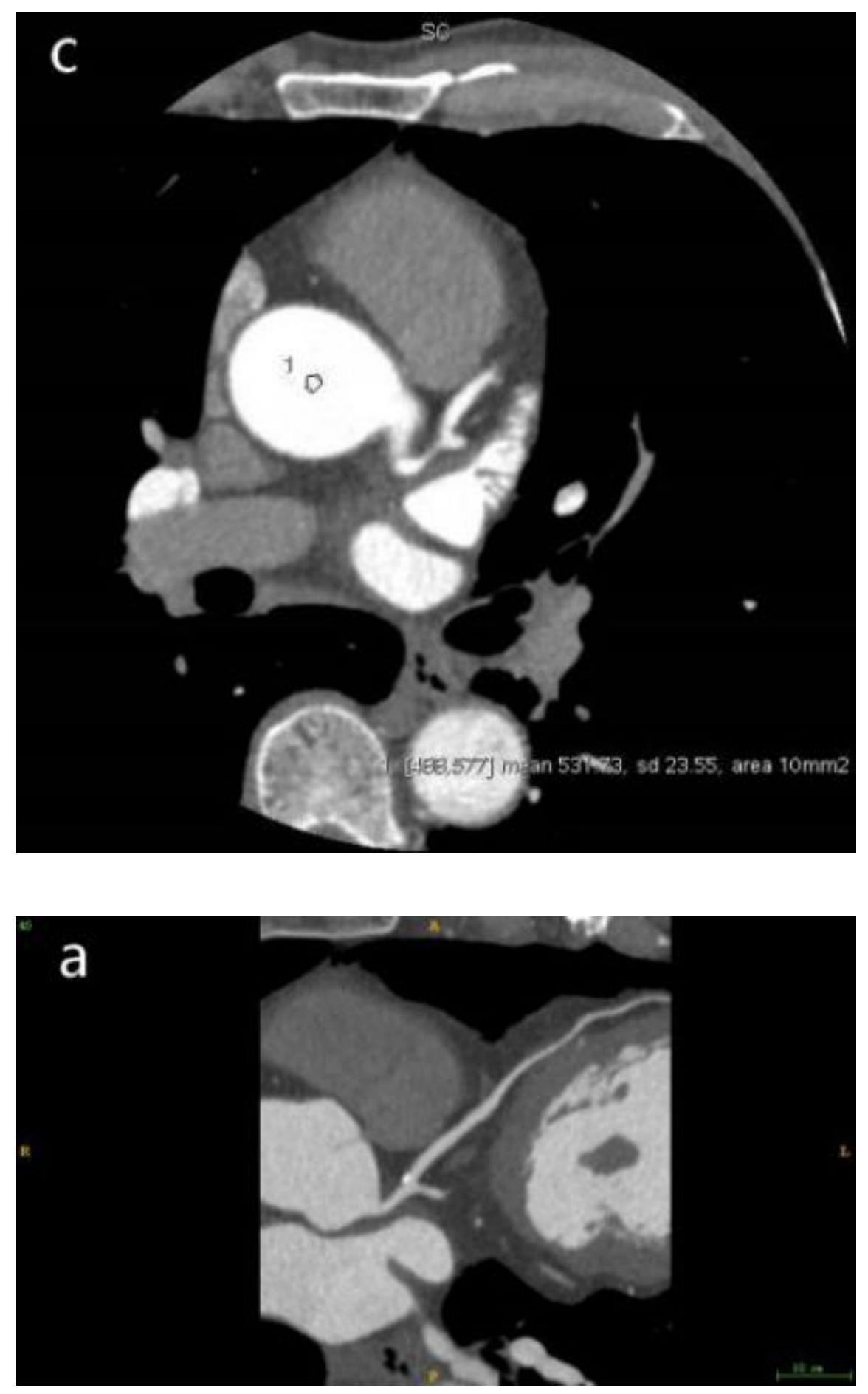

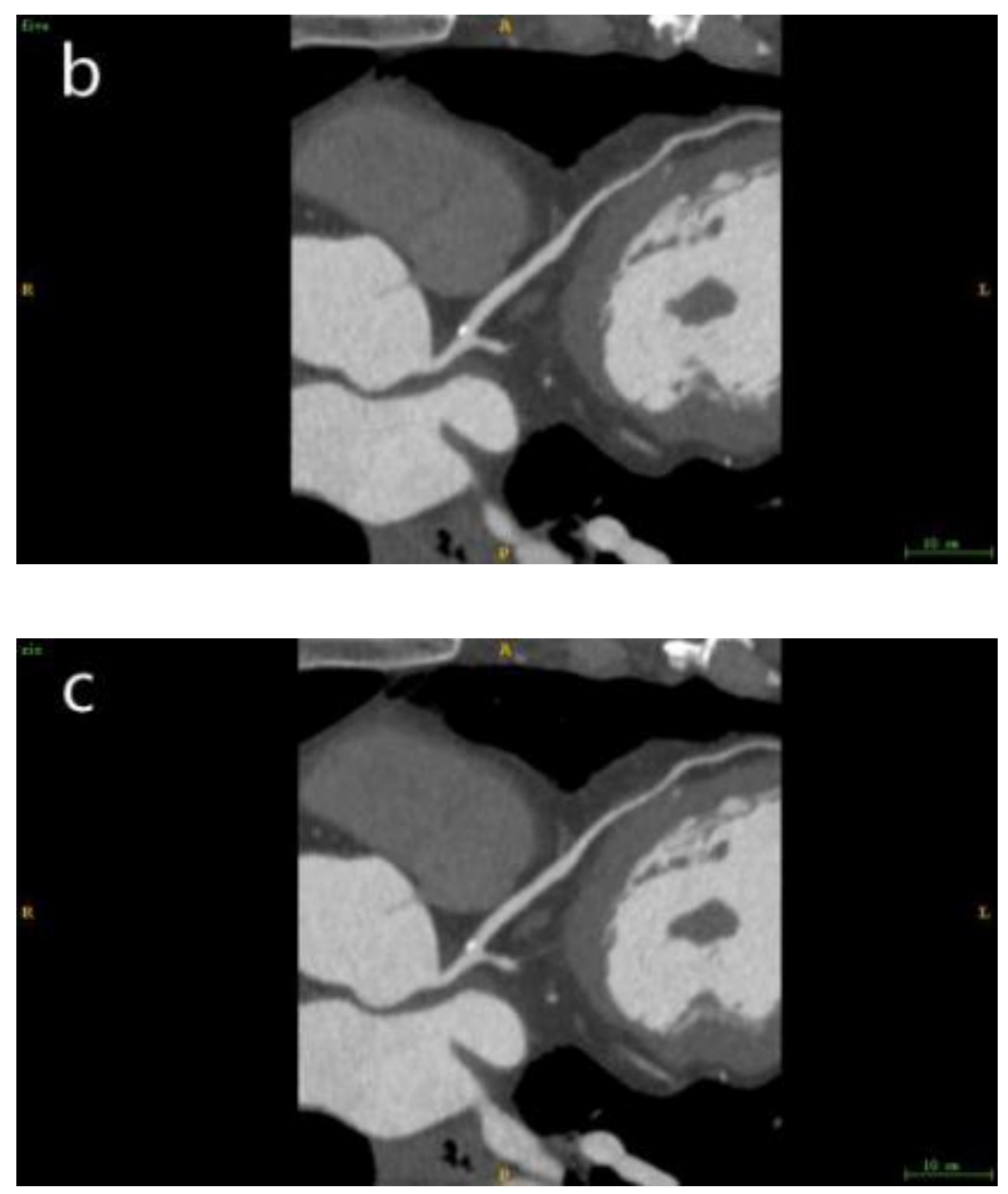


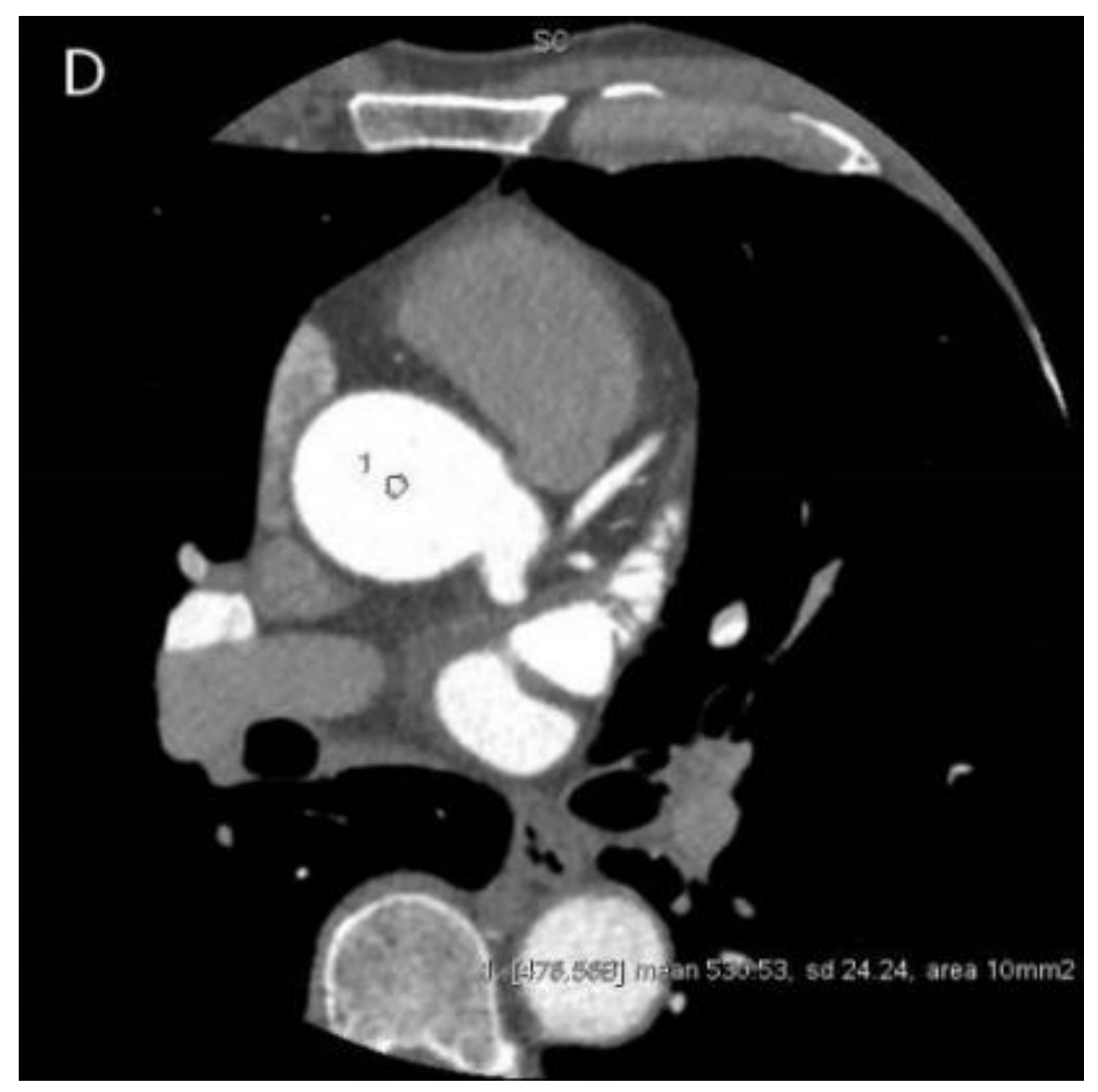




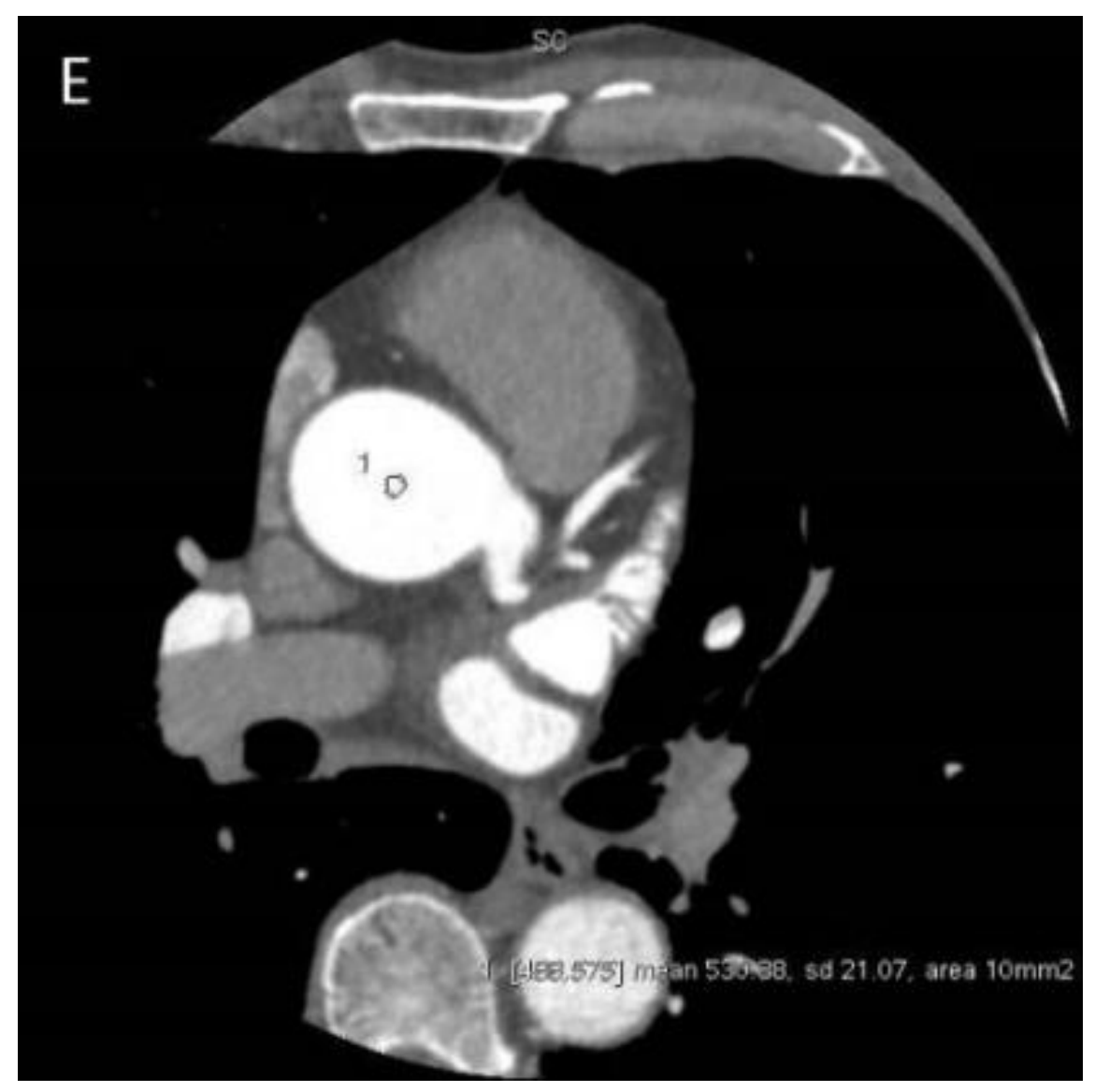



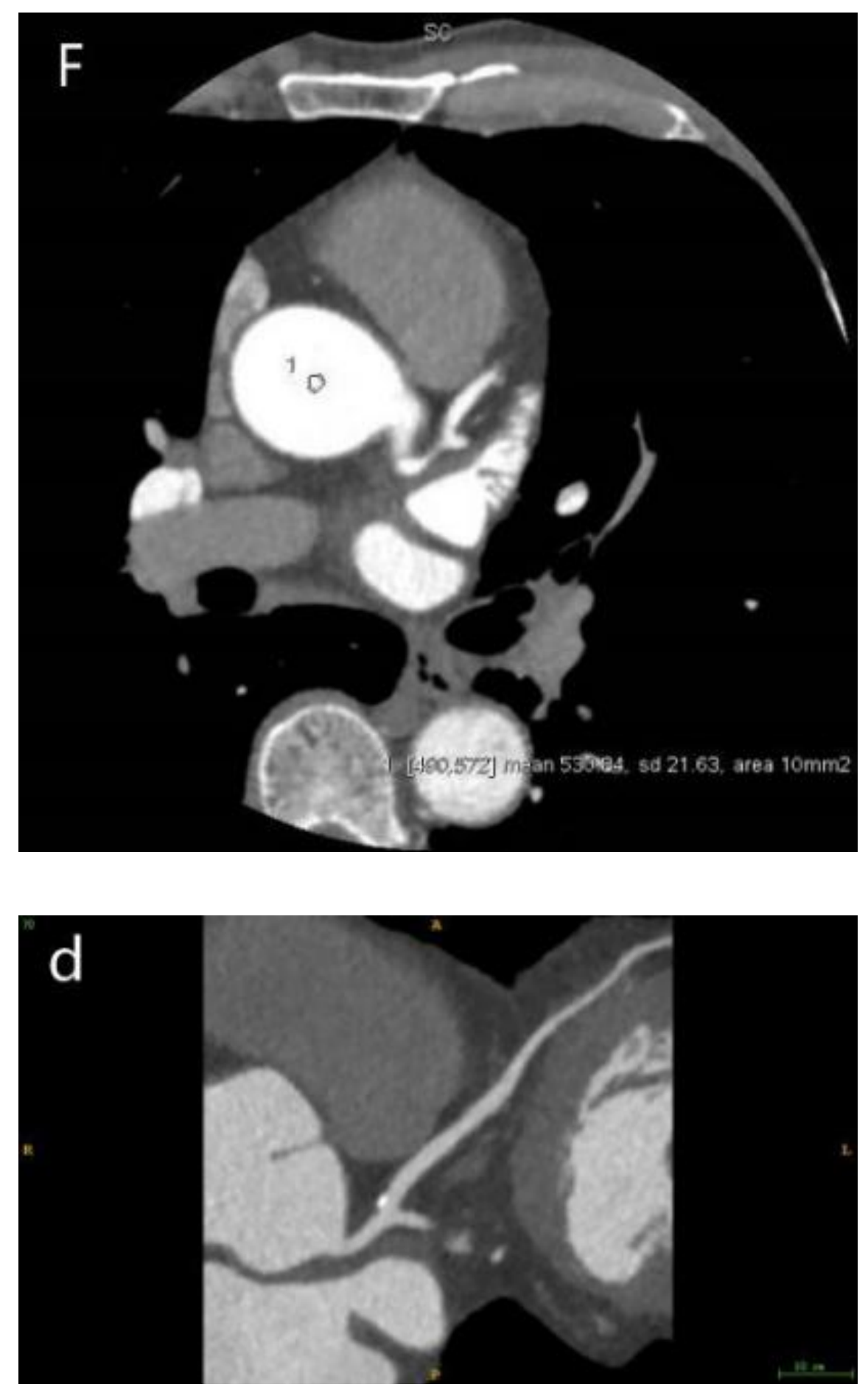

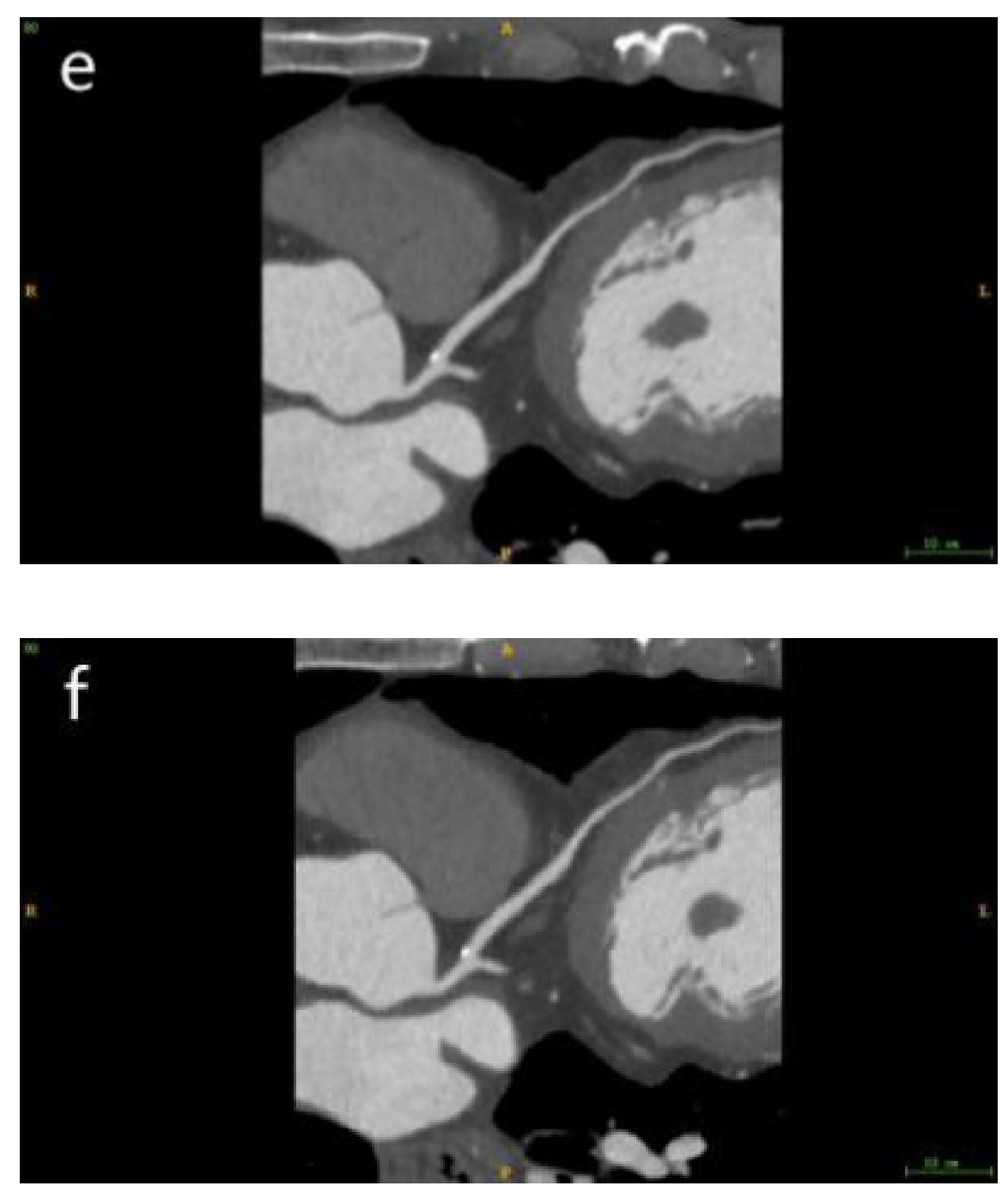

Figure 3. Coronary computed tomography angiography (CCTA) images acquired by different post-ASiR-V levels based on a 56 -year-old male patient with a body mass index of $25.7 \mathrm{~kg} / \mathrm{m}^{2}$. The CCTA images were scanned using $70 \%$ pre-ASiR-V combined with $40 \%$ post-ASiR-V (A), $50 \%$ post-ASiR-V (B), $60 \%$ post-ASiR$\mathrm{V}(\mathrm{C}), 70 \%$ post-ASiR-V (D), $80 \%$ post-ASiR-V (E), and $90 \%$ post-ASiR-V (F). With the percentage of post-ASiR-V increasing, the image noise decreased, but the computed tomography value did not significantly change. The subjective scores of images (A-F) were 3.42, 3.59, 3.64, 3.79, 3.98, and 3.57, respectively. Figure $\mathrm{E}$ had the highest score. Images a-f were the LAD images of the same patient with a $70 \%$ pre-ASiR-V level combined with $40 \%$ post-ASiR-V (A), $50 \%$ post-ASiR-V (B), $60 \%$ post- ASiR-V (C), $70 \%$ post-ASiR-V (D), $80 \%$ post-ASiR-V (E), and $90 \%$ post- $\mathrm{ASiR}-\mathrm{V}(\mathrm{F})$. Compared with the other images, the image quality of e ( $80 \%$ post-ASiR-V) was higher than that of $\mathrm{a}, \mathrm{b}, \mathrm{c}, \mathrm{d}$, and $\mathrm{f}$.

\section{REFERENCES}

[1] Akai H, Kiryu S, Shibata E, et al. Reducing CT radiation exposure with organ effective modulation: A retrospective clinical study. European journal of radiology. 2016;85(9):1569-73. Epub 2016/06/16. doi: 10.1016/j.ejrad.2016.06.008. PubMed PMID: 27501890.

[2] Fang T, Deng W, Law MW-M, et al. Comparison of image quality and radiation exposure between conventional imaging and gemstone spectral imaging in abdominal CT examination. Br J Radiol. 
2018;91(1088):20170448-. Epub 2018/06/01. doi: 10.1259/bjr.20170448. PubMed PMID: 29762057.

[3] Choi YJ, Lee JH, Yoon DH, et al. Effect of an Arm Traction Device on Image Quality and Radiation Exposure during Neck CT: A Prospective Study. AJNR Am J Neuroradiol. 2018;39(1):151-5. Epub 2017/11/09. doi: 10.3174/ajnr.A5418. PubMed PMID: 29122761.

[4] Weinman JP, Mirsky DM, Jensen AM, et al. Dual energy head CT to maintain image quality while reducing dose in pediatric patients. Clin Imaging. 2019;55:83-8. Epub 2019/02/07. doi: 10.1016/j.clinimag.2019.02.005. PubMed PMID: 30769223.

[5] Esser M, Hess S, Teufel M, et al. Radiation Dose Optimization in Pediatric Chest CT: Major Indicators of Dose Exposure in 1695 CT Scans over Seven Years. Rofo. 2018;190(12):1131-40. Epub 2018/10/11. doi: 10.1055/a-0628-7222. PubMed PMID: 30308691.

[6] Mousavi Gazafroudi SS, Tavakkoli MB, Moradi M, et al. Coronary CT angiography by modifying tube voltage and contrast medium concentration: Evaluation of image quality and radiation dose. Echocardiography. 2019;36(7):1391-6. Epub 2019/06/19. doi: 10.1111/echo.14410. PubMed PMID: 31215700.

[7] Lee J, Kim TH, Lee BK, et al. Diagnostic accuracy of low-radiation coronary computed tomography angiography with low tube voltage and knowledge-based model reconstruction. Sci Rep. 2019;9(1):1308-. doi: 10.1038/s41598-018-37870-3. PubMed PMID: 30718631.

[8] Lee HS, Suh YJ, Han K, et al. Effectiveness of automatic tube potential selection with tube current modulation in coronary CT angiography for obese patients: Comparison with a body mass index-based protocol using the propensity score matching method. PLoS One. 2018;13(1):e0190584-e. doi: 10.1371/journal.pone.0190584. PubMed PMID: 29304060.

[9] Papadakis AE, Damilakis J. Automatic Tube Current Modulation and Tube Voltage Selection in Pediatric Computed Tomography: A Phantom Study on Radiation Dose and Image Quality. Invest Radiol. 2019;54(5):265-72. doi: 10.1097/RLI.0000000000000537. PubMed PMID: 30562273.

[10] Yel I, Booz C, Albrecht MH, et al. Optimization of image quality and radiation dose using different cone-beam CT exposure parameters. European journal of radiology. 2019;116:68-75. Epub 2019/04/22. doi: 10.1016/j.ejrad.2019.04.005. PubMed PMID: 31153576.

[11] Ohno Y, Koyama H, Seki S, et al. Radiation dose reduction techniques for chest CT: Principles and clinical results. Eur J Radiol. 2019;111:93-103. Epub 2019/01/30. doi: 10.1016/j.ejrad.2018.12.017. PubMed PMID: 30691672.

[12] Solth A, Mukerji N, Strachan R. Reducing the radiation exposure from CT scanning in children with shunts: a nationwide survey and a departmental CT protocol. Br J Neurosurg. 2018;32(5):558-62. Epub 2018/07/18. doi: 10.1080/02688697.2018.1485874. PubMed PMID: 30019596.

[13] Leithner D, Gruber-Rouh T, Beeres M, et al. 90-kVp low-tube-voltage CT pulmonary angiography in combination with advanced modeled iterative reconstruction algorithm: effects on radiation dose, image quality and diagnostic accuracy for the detection of pulmonary embolism. Br J Radiol. 2018;91(1088):20180269. Epub 2018/05/25. doi: 10.1259/bjr.20180269. PubMed PMID: 29792729; PubMed Central PMCID: PMCPMC6209482.

[14] Barrington SF, Kluge R. FDG PET for therapy monitoring in Hodgkin and non-Hodgkin lymphomas. Eur J Nucl Med Mol Imaging. 2017;44(Suppl 1):97-110. Epub 2017/04/14. doi: 10.1007/s00259-017-3690-8. PubMed PMID: 28411336.

[15] Trattner S, Halliburton S, Thompson CM, et al. Cardiac-Specific Conversion Factors to Estimate Radiation Effective Dose From Dose-Length Product in Computed Tomography. JACC Cardiovascular imaging. 2018;11(1):64-74. Epub 2017/08/22. doi: 10.1016/j.jcmg.2017.06.006. PubMed PMID: 28823748; PubMed Central PMCID: PMCPMC5756125. 
[16] Richards CE, Obaid DR. Low-Dose Radiation Advances in Coronary Computed Tomography Angiography in the Diagnosis of Coronary Artery Disease. Curr Cardiol Rev. 2019;15(4):304-15. Epub 2019/02/27. doi: 10.2174/1573403X15666190222163737. PubMed PMID: 30806322.

[17] Rudziński PN, Kruk M, Kepka C, et al. The value of Coronary Artery computed Tomography as the first-line anatomical test for stable patients with indications for invasive angiography due to suspected Coronary Artery Disease: CAT-CAD randomized trial. J Cardiovasc Comput Tomogr. 2018;12(6):472-9. Epub 2018/09/04. doi: 10.1016/j.jcct.2018.08.004. PubMed PMID: 30201310.

[18] Lin ZX, Zhou CS, Schoepf UJ, et al. Coronary CT angiography radiation dose trends: A 10-year analysis to develop institutional diagnostic reference levels. Eur J Radiol. 2019;113:140-7. Epub 2019/04/01. doi: 10.1016/j.ejrad.2019.02.012. PubMed PMID: 30927938.

[19] Rampinelli C, De Marco P, Origgi D, et al. Exposure to low dose computed tomography for lung cancer screening and risk of cancer: secondary analysis of trial data and risk-benefit analysis. BMJ. 2017;356:j347. Epub 2017/02/10. doi: 10.1136/bmj.j347. PubMed PMID: 28179230; PubMed Central PMCID: PMCPMC5421449.

[20] Ohira S, Kanayama N, Wada K, et al. A Third-Generation Adaptive Statistical Iterative Reconstruction for Contrast-Enhanced 4-Dimensional Dual-Energy Computed Tomography for Pancreatic Cancer. J Comput Assist Tomogr. 2019:10.1097/RCT.0000000000000942. doi: 10.1097/RCT.0000000000000942. PubMed PMID: 31738200.

[21] De Marco P, Origgi D. New adaptive statistical iterative reconstruction ASiR-V: Assessment of noise performance in comparison to ASiR. J Appl Clin Med Phys. 2018;19(2):275-86. Epub 2018/01/24. doi: 10.1002/acm2.12253. PubMed PMID: 29363260.

[22] Pontone G, Muscogiuri G, Andreini D, et al. Impact of a New Adaptive Statistical Iterative Reconstruction (ASIR)-V Algorithm on Image Quality in Coronary Computed Tomography Angiography. Acad Radiol. 2018;25(10):1305-13. Epub 2018/03/27. doi: 10.1016/j.acra.2018.02.009. PubMed PMID: 29602723.

[23] Wang XP, Hou P, Lü PJ, et al. Application of adaptive statistical iterative reconstruction V (ASIR$\mathrm{V}$ ) in contrast-enhanced abdominal scanning with low-dose for liver cirrhosis. Zhonghua Yi Xue Za Zhi. 2019;99(27):2124-9. doi: 10.3760/cma.j.issn.0376-2491.2019.27.006. PubMed PMID: 31315384.

[24] Park C, Choo KS, Kim JH, et al. Image Quality and Radiation Dose in CT Venography Using ModelBased Iterative Reconstruction at $80 \mathrm{kVp}$ versus Adaptive Statistical Iterative Reconstruction-V at $70 \mathrm{kVp}$. Korean J Radiol. 2019;20(7):1167-75. doi: 10.3348/kjr.2018.0897. PubMed PMID: 31270980.

[25] Laurent G, Villani N, Hossu G, et al. Full model-based iterative reconstruction (MBIR) in abdominal CT increases objective image quality, but decreases subjective acceptance. Eur Radiol. 2019;29(8):4016-25. Epub 2019/01/30. doi: 10.1007/s00330-018-5988-8. PubMed PMID: 30701327.

[26] Wang HX, Lü PJ, Yue SW, et al. Combined use of wide-detector and adaptive statistical iterative reconstruction-V technique in abdominal $\mathrm{CT}$ with low radiation dose. Zhonghua Yi Xue Za Zhi. 2017;97(45):3567-72. doi: 10.3760/cma.j.issn.0376-2491.2017.45.011. PubMed PMID: 29275597.

[27] Elmokadem AH, Ibrahim EA, Gouda WA, et al. Whole-Body Computed Tomography Using LowDose Biphasic Injection Protocol With Adaptive Statistical Iterative Reconstruction V: Assessment of Dose Reduction and Image Quality in Trauma Patients. J Comput Assist Tomogr. 2019;43(6):870-6. doi: 10.1097/RCT.0000000000000907. PubMed PMID: 31453974.

[28] Chen L-H, Jin C, Li J-Y, et al. Image quality comparison of two adaptive statistical iterative reconstruction (ASiR, ASiR-V) algorithms and filtered back projection in routine liver CT. Br J Radiol. 2018;91(1088):20170655-. Epub 2018/06/06. doi: 10.1259/bjr.20170655. PubMed PMID: 29848018.

[29] Goodenberger MH, Wagner-Bartak NA, Gupta S, et al. Computed Tomography Image Quality Evaluation of a New Iterative Reconstruction Algorithm in the Abdomen (Adaptive Statistical Iterative 
Reconstruction-V) a Comparison With Model-Based Iterative Reconstruction, Adaptive Statistical Iterative Reconstruction, and Filtered Back Projection Reconstructions. J Comput Assist Tomogr. 2018;42(2):184-90. doi: 10.1097/RCT.0000000000000666. PubMed PMID: 28806318.

[30] Rotzinger DC, Racine D, Beigelman-Aubry C, et al. Task-Based Model Observer Assessment of A Partial Model-Based Iterative Reconstruction Algorithm in Thoracic Oncologic Multidetector CT. Sci Rep. 2018;8(1):17734-. doi: 10.1038/s41598-018-36045-4. PubMed PMID: 30531988.

[31] Tang H, Yu N, Jia Y, et al. Assessment of noise reduction potential and image quality improvement of a new generation adaptive statistical iterative reconstruction (ASIR-V) in chest CT. Br J Radiol. 2018;91(1081):20170521-. Epub 2017/11/16. doi: 10.1259/bjr.20170521. PubMed PMID: 29076347.

\section{Hosted file}

Figure.docx available at https://authorea.com/users/317720/articles/447812-comparison-ofimage-quality-and-radiation-dose-using-different-pre-asir-v-and-post-asir-v-levels-incoronary-computed-tomography-angiography

\section{Hosted file}

Table of Contents.docx available at https://authorea.com/users/317720/articles/447812comparison-of-image-quality-and-radiation-dose-using-different-pre-asir-v-and-post-asirv-levels-in-coronary-computed-tomography-angiography 